\title{
A statistical analysis plan for the Adjunctive Corticosteroids for Tuberculous meningitis in HIV-positive adults (ACT HIV) clinical trial [version 1; peer review: 3 approved]
}

Joseph Donovan (D1-3, Trinh Dong Huu Khanh1,2, Guy E. Thwaites (D1,2, Ronald B. Geskus (D)1,2, ACT HIV investigators

${ }^{1}$ Oxford University Clinical Research Unit, Centre for Tropical Medicine, Ho Chi Minh City, Vietnam

${ }^{2}$ Centre for Tropical Medicine and Global Health, Nuffield Department of Medicine, University of Oxford, Oxford, UK

${ }^{3}$ London School of Hygiene and Tropical Medicine, London, UK

V1 First published: 19 Oct 2021, 6:280

https://doi.org/10.12688/wellcomeopenres.17154.1

Latest published: $07 \mathrm{Jul} 2022,6: 280$

https://doi.org/10.12688/wellcomeopenres.17154.2

\section{Abstract}

TBM is the most severe form of tuberculosis. Clinical trial data are required to provide an evidence base for adjunctive dexamethasone in HIV-positive individuals with TBM, and to guide clinical practice. This document details the planned analyses at 12 months post randomisation for the ACT HIV clinical trial (NCT03092817); 'a randomised double-blind placebo-controlled trial of adjunctive dexamethasone for the treatment of HIV co-infected adults with tuberculous meningitis (TBM)'. The primary endpoint of the ACT HIV trial is death (from any cause) over the first 12 months after randomisation. This statistical analysis plan expands upon and updates the analysis plan outlined in the published study protocol.

\section{Keywords}

Tuberculous meningitis, human immunodeficiency virus, corticosteroids, clinical trial, analysis plan

11001 This article is included in the Oxford University oucru
Clinical Research Unit (OUCRU) gateway.

\section{Open Peer Review}

Approval Status

1

2 3

\section{version 2}

(revision)

07 Jul 2022

version 1

19 Oct 2021
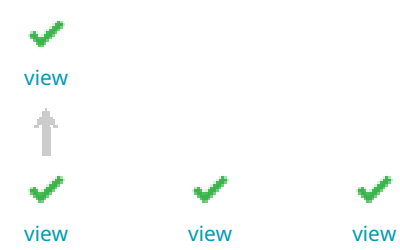

1. Nico (Nicolaas) Nagelkerke (iD), University of Toronto, Toronto, Canada

2. Ravindra Kumar Garg (ID), King George's

Medical University, Lucknow, India

Hardeep Singh Malhotra (D), King George's

Medical University, Lucknow, India

3. Peter Mwaba, Lusaka Apex Medical

University, Lusaka, Zambia

Any reports and responses or comments on the article can be found at the end of the article. 
Corresponding author: Joseph Donovan (jdonovan@oucru.org)

Author roles: Donovan J: Investigation, Methodology, Project Administration, Writing - Original Draft Preparation, Writing - Review \& Editing; Khanh TDH: Data Curation, Methodology, Software, Writing - Review \& Editing; Thwaites GE: Conceptualization, Funding Acquisition, Methodology, Supervision, Writing - Original Draft Preparation, Writing - Review \& Editing; Geskus RB: Formal Analysis, Methodology, Software, Supervision, Writing - Original Draft Preparation, Writing - Review \& Editing;

Competing interests: No competing interests were disclosed.

Grant information: The ACT HIV trial is supported by Wellcome [110179], an Investigator Award to Professor Guy Thwaites. The funders had no role in study design, data collection and analysis, decision to publish, or preparation of the manuscript.

Copyright: @ 2021 Donovan J et al. This is an open access article distributed under the terms of the Creative Commons Attribution License, which permits unrestricted use, distribution, and reproduction in any medium, provided the original work is properly cited.

How to cite this article: Donovan J, Khanh TDH, Thwaites GE et al. A statistical analysis plan for the Adjunctive Corticosteroids for Tuberculous meningitis in HIV-positive adults (ACT HIV) clinical trial [version 1; peer review: 3 approved] Wellcome Open Research 2021, 6:280 https://doi.org/10.12688/wellcomeopenres.17154.1

First published: 19 Oct 2021, 6:280 https://doi.org/10.12688/wellcomeopenres.17154.1 


\section{Scope of document}

This document details the planned analyses at 12 months post randomisation for the ACT HIV clinical trial (NCT03092817); 'a randomised double-blind placebo-controlled trial of adjunctive dexamethasone for the treatment of HIV co-infected adults with tuberculous meningitis (TBM)'. A further analysis will follow at 24 months post randomisation. This statistical analysis plan expands upon and updates the analysis plan outlined in the published study protocol ${ }^{1}$.

\section{Background and rationale for study}

The ACT HIV clinical trial is a randomised double-blind placebo-controlled trial of adjunctive dexamethasone for the treatment of tuberculous meningitis (TBM) in HIV-positive adults. TBM is the most severe form of tuberculosis, with mortality approaching $50 \%$ in people living with $\mathrm{HIV}^{2-5}$ despite the best available appropriate anti-TB chemotherapy.

TBM develops when Mycobacterium tuberculosis, initially acquired via the respiratory route, disseminates via the blood to form a secondary focus in the brain. Rupture of this secondary focus into the subarachnoid space results in TBM, with the consequent host inflammatory response being important for bacterial killing, but also responsible for the pathological complications and often-fatal consequences of the infection.

Adjunctive anti-inflammatory corticosteroid therapy, which may control excessive host inflammation, has long been considered to have a role in TBM treatment ${ }^{6}$. In 2004, a landmark clinical trial in Vietnam demonstrated that a 6-8 week (duration dependent on Modified Research Council [MRC] TBM severity grade) tapering course of dexamethasone reduced mortality in adults and adolescents with $\mathrm{TBM}^{4}$. In this study, only 98/545 (18.0\%) individuals were HIV positive, which was too small to determine if dexamethasone benefited this important sub-group of patients. No other trials have been published since 2004 that examine whether corticosteroids improve outcomes from TBM in HIV-positive adults.

How dexamethasone might confer its clinical benefit on those with TBM remains uncertain. A recent study of cerebrospinal fluid (CSF) cytokines in TBM suggested that HIVpositive individuals have higher cytokine concentrations than HIV-negative individuals ${ }^{7}$. Whether adjunctive dexamethasone is more or less likely to be beneficial in HIV-positive individuals with TBM is not known. There are an estimated 100,000 cases of TBM globally each year ${ }^{8}$, many associated with HIV. Clinical trial data are required to provide an evidence base for adjunctive dexamethasone in HIV-positive individuals with TBM, and to guide clinical practice.

\section{Structure and status of trial}

The ACT HIV trial commenced recruitment on $25^{\text {th }}$ May 2017. By April 29 2021 , the predefined sample size of 520 adults with TBM and HIV co-infection had been enrolled from four hospitals in Vietnam and Indonesia: the Hospital for Tropical Diseases (HTD) and Pham Ngoc Thach Hospital for Tuberculosis and Lung Disease (PNT) in Ho Chi Minh
City, Vietnam, and Cipto Mangunkusumo Hospital and Persahabatan Hospital in Jakarta, Indonesia. Detailed enrolment criteria for ACT HIV, including consent and ethical approvals, are described in the published trial protocol ${ }^{1}$.

Once enrolled, study participants were randomised to dexamethasone or placebo (a double-blinded allocation), with this intervention termed 'study drug'. Randomisation was stratified by TBM MRC severity score and by hospital. Participants with MRC grade 1 TBM received a 6-week tapering course of study drug, whereas participants with MRC grades 2 or 3 received an 8-week tapering course of study drug. Study drug regimens are shown in Table 1. Participants then underwent clinical assessments at baseline, at days 3, 7, 10, 14, 21, and 30, monthly until month 12 , and then 3-monthly until 24 months. Baseline assessment included blood tests, chest $\mathrm{X}$-ray, lumbar puncture, and brain imaging.

ACT HIV has numerous sub-studies, as described in the protocol $^{1}$. Key follow up outcomes include survival, disability, and severe adverse events; especially neurological events, events requiring corticosteroids, HIV-associated complications (malignancies, immune reconstitution inflammatory syndrome [IRIS]), and adverse events considered related to dexamethasone.

\section{Trial endpoints}

\section{Primary endpoint}

The primary endpoint of the ACT HIV trial is death (from any cause) over the first 12 months after randomisation.

\section{Secondary endpoints}

The secondary endpoints of the ACT HIV trial are as follows:

1. Neurological disability at 12 months from randomisation

2. First new neurological event or death over the first 12 months after randomisation

Table 1. Study drug treatment regimen following randomisation.

\begin{tabular}{|l|l|l|}
\hline & $\begin{array}{l}\text { MRC Grade I } \\
\text { Daily dexamethasone } \\
\text { dose/route }\end{array}$ & $\begin{array}{l}\text { MRC Grades II and III } \\
\text { Daily dexamethasone } \\
\text { dose/route }\end{array}$ \\
\hline Week 1 & $0.3 \mathrm{mg} / \mathrm{kg} / 24 \mathrm{hrs} \mathrm{IV}$ & $0.4 \mathrm{mg} / \mathrm{kg} / 24 \mathrm{hrs} \mathrm{IV}$ \\
\hline Week 2 & $0.2 \mathrm{mg} / \mathrm{kg} / 24 \mathrm{hrs} \mathrm{IV}$ & $0.3 \mathrm{mg} / \mathrm{kg} / 24 \mathrm{hrs} \mathrm{IV}$ \\
\hline Week 3 & $0.1 \mathrm{mg} / \mathrm{kg} / 24 \mathrm{hrs} \mathrm{IV}$ & $0.2 \mathrm{mg} / \mathrm{kg} / 24 \mathrm{hrs} \mathrm{IV}$ \\
\hline Week 4 & $3 \mathrm{mg} / 24 \mathrm{hrs}$ oral & $0.1 \mathrm{mg} / \mathrm{kg} / 24 \mathrm{hrs} \mathrm{IV}$ \\
\hline Week 5 & $2 \mathrm{mg} / 24 \mathrm{hrs}$ oral & $4 \mathrm{mg} / 24 \mathrm{hrs}$ oral \\
\hline Week 6 & $1 \mathrm{mg} / 24 \mathrm{hrs}$ oral & $3 \mathrm{mg} / 24 \mathrm{hrs}$ oral \\
\hline Week 7 & Stop & $2 \mathrm{mg} / 24 \mathrm{hrs}$ oral \\
\hline Week 8 & & $1 \mathrm{mg} / 24 \mathrm{hrs}$ oral \\
\hline
\end{tabular}

IV=intravenous. MRC=Modified Research Council. 
3. Neurological IRIS events over the first 6 months after randomisation

4. New AIDS-defining event or death over the first 12 months after randomisation

\section{HIV-associated malignancy by 12 months from randomisation}

6. Use of open-label corticosteroid treatment for any reason over the first 12 months after randomisation

7. Requirement for shunt surgery by 12 months

8. Any serious adverse events reported by 12 months from randomisation

\section{Individuals included in analysis Primary analysis}

The primary analysis includes all randomised participants. As such, an intention-to-treat (ITT) analysis will be performed for the primary and secondary endpoints. Participants will remain included in this analysis even if no study drug was received after randomisation.

\section{Per protocol analysis}

The per protocol (PP) analysis includes all randomised patients with the exception of those with a final diagnosis other than TBM (confirmed by microbiology, serology, or histopathology), and those who received less than 7 days of administration of the randomised study drug for reasons other than death, or less than 30 days of anti-tuberculosis drugs for any reason other than death. A per protocol analysis will be performed for all primary and secondary endpoints.

\section{Statistical software}

Data will be analysed using the program $R^{9}$, using the most up to date version available at the time of final analysis.

\section{Baseline characteristics}

Definition: The following baseline characteristics will be summarised by treatment arm for ITT and PP analyses: age, sex, country, site, diagnostic category (definite, probable, possible, or not TBM by Marais criteria ${ }^{10}$ ), history of previous tuberculosis treatment, chest X-ray findings (no TB/miliary TB/pulmonary TB), enrolment MRC TBM grade, enrolment Glasgow coma score (GCS), weight ( $\mathrm{kg}$ ), duration of symptoms, cranial nerve palsy, hemiplegia, para/tetraplegia, urinary retention, history of diabetes, $\mathrm{HbA} 1 \mathrm{c}$, history of intravenous drug use, hepatitis B sAg positivity, hepatitis C Ab positivity, alanine aminotransferase (ALT), bilirubin, full blood count (haemoglobin, white cell count, platelets), plasma sodium, leukotriene A4 hydrolase $(L T A 4 H)$ genotype $(\mathrm{CC} / \mathrm{CT} / \mathrm{TT})$, routine CSF parameters (opening pressure, total leucocytes, total neutrophils, total lymphocytes, protein, blood:CSF glucose ratio, Ziehl-Neelsen stain, Gene Xpert MTB/RIF, Gene Xpert MTB/RIF Ultra, mycobacterial culture), duration of antituberculosis chemotherapy (days) before enrolment, enrolment anti-TB regimen, anti-TB drug susceptibility results in culture-confirmed sub-group (multi-drug resistant [MDR] TB or rifampicin mono-resistant $\mathrm{TB}$, isoniazid resistant non-MDR, pre-extensively drug resistant [XDR] TB, XDR TB, no or other resistance), HIV infection (new or known diagnosis), antiretroviral therapy (ART) treatment at enrolment (ART naïve, $\leq 3$ months of ART, $>3$ months of ART) and time to starting ART (if ART naïve), enrolment CD4 count (median values and categories [<50, 51-100, 101-200, >200]), HIV viral load. These will be described in a baseline variable table (Table 2).

Analysis: Baseline characteristics will be summarised as median (lower and upper quartiles) for continuous data and frequency (percentage) for categorical data. The amount of missing data for each baseline characteristic will also be displayed. We will perform multiple imputation of a variable in case of $>5 \%$ missing values, and it is assumed that data are missing at random. Otherwise, a complete case analysis will be performed.

\section{Use of the uniform case definition diagnostic score}

The published TBM diagnostic score ${ }^{10}$ will be used and subjects will be categorised as 'definite', 'probable', 'possible', or 'not TBM'. Participants will only be categorised as 'not TBM' if they have a confirmed alternative diagnosis (alternative to TBM) or they recovered without TB drugs.

\section{Primary endpoint}

The primary endpoint of this trial is death over the first 12 months after randomisation. The primary analysis is a Cox proportional hazards regression model with treatment as the only covariate. Since TBM MRC severity grade at enrolment (I, II, or III) is an important risk factor for mortality and was used as stratification variable in the randomisation, we will additionally perform a secondary analysis with MRC grade added as stratum variable. The proportional hazards assumption will be formally tested based on scaled Schoenfeld residuals and visually assessed by a plot of the scaled Schoenfeld residuals versus transformed time. Deaths, by study drug arm, and the results of the analyses will be shown as per Table 3 and Table 4.

Survivors known to be alive at 12 months will be censored at that time-point and subjects who withdrew or were lost to follow-up before 12 months will be censored at the date they were last known to be alive. Subjects who withdrew or were lost to follow-up before 12 months are estimated to be less than $5 \%$ of enrolled participants.

The homogeneity of the treatment effect on overall survival across subgroups will be assessed by formal tests of interaction between treatment and the following pre-defined grouping variables: TBM MRC severity grade at enrolment (I, II, or III), diagnostic category (definite, probable, possible), $L T A 4 H$ genotype (CC/CT/TT), drug resistance pattern (MDR TB or rifampicin mono-resistant $\mathrm{TB}$, isoniazid resistant non-MDR, no or other resistance), ART status at enrolment (ART naïve, $\leq 3$ months of ART, $>3$ months of ART), and CD4 cell count at enrolment $(<50,51-100,101-200,>200)$. We will estimate 
Table 2. Baseline characteristics template table.

\begin{tabular}{|c|c|c|c|c|}
\hline \multirow[t]{2}{*}{ Characteristic } & \multicolumn{2}{|c|}{$\begin{array}{l}\text { Dexamethasone } \\
(\mathrm{N}=\mathrm{XX})\end{array}$} & \multicolumn{2}{|c|}{$\begin{array}{l}\text { Placebo } \\
(\mathrm{N}=\mathrm{XX})\end{array}$} \\
\hline & $\mathbf{N}$ & $\begin{array}{l}\text { Summary } \\
\text { statistic }\end{array}$ & $\mathbf{N}$ & $\begin{array}{l}\text { Summary } \\
\text { statistic }\end{array}$ \\
\hline Age (years) & $X X$ & $X X(X X, X X)$ & $x X$ & $X X(X X, X X)$ \\
\hline Sex-male & $x X$ & $X X(X X \%)$ & $X X$ & $X X(X X \%)$ \\
\hline Country & $x X$ & $X X(X X \%)$ & $X X$ & $X X(X X \%)$ \\
\hline $\begin{array}{l}\text { Site } \\
\text { - HTD } \\
\text { - PNT } \\
\text { - Cipto Mangunkusumo } \\
\text { - Persahabatan }\end{array}$ & $\begin{array}{l}X X \\
X X \\
X X \\
X X\end{array}$ & $\begin{array}{l}X X(X X \%) \\
X X(X X \%) \\
X X(X X \%) \\
X X(X X \%)\end{array}$ & $\begin{array}{l}X X \\
X X \\
X X \\
X X\end{array}$ & $\begin{array}{l}X X(X X \%) \\
X X(X X \%) \\
X X(X X \%) \\
X X(X X \%)\end{array}$ \\
\hline $\begin{array}{l}\text { Diagnostic category } \\
\text { - Definite TBM } \\
\text { - Probable TBM } \\
\text { - Possible TBM } \\
\text { - Not TBM }\end{array}$ & $\begin{array}{l}X X \\
X X \\
X X \\
X X\end{array}$ & $\begin{array}{l}X X(X X \%) \\
X X(X X \%) \\
X X(X X \%) \\
X X(X X \%)\end{array}$ & $\begin{array}{l}X X \\
X X \\
X X \\
X X\end{array}$ & $\begin{array}{l}X X(X X \%) \\
X X(X X \%) \\
X X(X X \%) \\
X X(X X \%)\end{array}$ \\
\hline Previous tuberculosis treatment & $X X$ & $X X(X X \%)$ & $X X$ & $X X(X X \%)$ \\
\hline $\begin{array}{l}\text { Chest X-ray findings } \\
\text { - No tuberculosis } \\
\text { - Miliary tuberculosis } \\
\text { - Pulmonary tuberculosis }\end{array}$ & $\begin{array}{l}X X \\
X X \\
X X\end{array}$ & $\begin{array}{l}X X(X X \%) \\
X X(X X \%) \\
X X(X X \%)\end{array}$ & $\begin{array}{l}X X \\
X X \\
X X\end{array}$ & $\begin{array}{l}X X(X X \%) \\
X X(X X \%) \\
X X(X X \%)\end{array}$ \\
\hline $\begin{array}{l}\text { Modified MRC grade } \\
\text { - Grade I } \\
\text { - Grade II } \\
\text { - Grade III }\end{array}$ & $\begin{array}{l}X X \\
X X \\
X X\end{array}$ & $\begin{array}{l}X X(X X \%) \\
X X(X X \%) \\
X X(X X \%)\end{array}$ & $\begin{array}{l}X X \\
X X \\
X X\end{array}$ & $\begin{array}{l}X X(X X \%) \\
X X(X X \%) \\
X X(X X \%)\end{array}$ \\
\hline Glasgow coma score & $X X$ & $X X(X X, X X)$ & $X X$ & $X X(X X, X X)$ \\
\hline Weight (kg) & $x X$ & $X X(X X, X X)$ & $X X$ & $X X(X X, X X)$ \\
\hline Duration of symptoms (days) & $x x$ & $X X(X X, X X)$ & $x X$ & $X X(X X, X X)$ \\
\hline $\begin{array}{l}\text { Neurological signs } \\
\text { - Cranial nerve palsy } \\
\text { - Hemiplegia } \\
\text { - Paraplegia/tetraplegia } \\
\text { - Urinary retention }\end{array}$ & $\begin{array}{l}X X \\
X X \\
X X \\
X X\end{array}$ & $\begin{array}{l}X X(X X \%) \\
X X(X X \%) \\
X X(X X \%) \\
X X(X X \%)\end{array}$ & $\begin{array}{l}X X \\
X X \\
X X \\
X X\end{array}$ & $\begin{array}{l}X X(X X \%) \\
X X(X X \%) \\
X X(X X \%) \\
X X(X X \%)\end{array}$ \\
\hline History of diabetes & $X X$ & $X X(X X \%)$ & $X X$ & $X X(X X \%)$ \\
\hline HbA1c (\%) & $x X$ & $X X(X X, X X)$ & $X X$ & $X X(X X, X X)$ \\
\hline History of intravenous drug use & $x X$ & $X X(X X \%)$ & $x X$ & $X X(X X \%)$ \\
\hline Hepatitis B sAg positivity, & $X X$ & $X X(X X \%)$ & $X X$ & $X X(X X \%)$ \\
\hline Hepatitis C Ab positivity & $X X$ & $X X(X X \%)$ & $X X$ & $X X(X X \%)$ \\
\hline Alanine aminotransferase (ALT) (IU/L) & $X X$ & $X X(X X, X X)$ & $X X$ & $X X(X X, X X)$ \\
\hline Bilirubin ( $\mu \mathrm{mol} / \mathrm{L})$ & $x X$ & $X X(X X, X X)$ & $x X$ & $X X(X X, X X)$ \\
\hline
\end{tabular}




\begin{tabular}{|c|c|c|c|c|}
\hline \multirow[t]{2}{*}{ Characteristic } & \multicolumn{2}{|c|}{$\begin{array}{l}\text { Dexamethasone } \\
(\mathrm{N}=\mathrm{XX})\end{array}$} & \multicolumn{2}{|c|}{$\begin{array}{l}\text { Placebo } \\
(\mathrm{N}=\mathrm{XX})\end{array}$} \\
\hline & $\mathbf{N}$ & $\begin{array}{l}\text { Summary } \\
\text { statistic }\end{array}$ & $\mathbf{N}$ & $\begin{array}{l}\text { Summary } \\
\text { statistic }\end{array}$ \\
\hline $\begin{array}{l}\text { Full blood count } \\
\text { - Haemoglobin }(\mathrm{g} / \mathrm{dL}) \\
\text { - White cell count }\left(\times 10^{3} / \mu \mathrm{L}\right) \\
\text { - Platelets }\left(\times 10^{3} / \mu \mathrm{L}\right)\end{array}$ & $\begin{array}{l}X X \\
X X \\
X X\end{array}$ & $\begin{array}{l}X X(X X, X X) \\
X X(X X, X X) \\
X X(X X, X X)\end{array}$ & $\begin{array}{l}X X \\
X X \\
X X\end{array}$ & $\begin{array}{l}X X(X X, X X) \\
X X(X X, X X) \\
X X(X X, X X)\end{array}$ \\
\hline \multicolumn{5}{|l|}{ Plasma sodium (mmol/L) } \\
\hline $\begin{array}{l}\text { Leukotriene A4 hydrolase } \\
\text { - TT } \\
\text { - CT } \\
\text { - CC }\end{array}$ & $\begin{array}{l}X X \\
X X \\
X X\end{array}$ & $\begin{array}{l}X X(X X \%) \\
X X(X X \%) \\
X X(X X \%)\end{array}$ & $\begin{array}{l}X X \\
X X \\
X X\end{array}$ & $\begin{array}{l}X X(X X \%) \\
X X(X X \%) \\
X X(X X \%)\end{array}$ \\
\hline 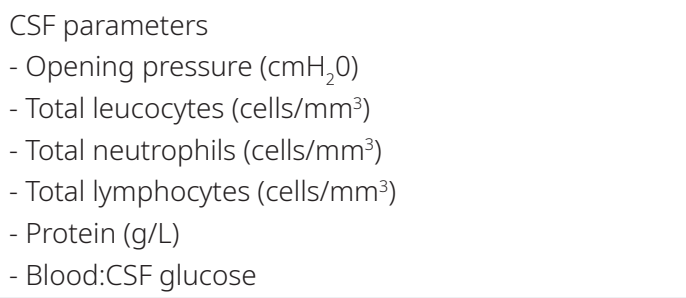 & $\begin{array}{l}X X \\
X X \\
X X \\
X X \\
X X \\
X X\end{array}$ & $\begin{array}{l}X X(X X, X X) \\
X X(X X, X X) \\
X X(X X, X X) \\
X X(X X, X X) \\
X X(X X, X X) \\
X X(X X, X X)\end{array}$ & $\begin{array}{l}X X \\
X X \\
X X \\
X X \\
X X \\
X X\end{array}$ & $\begin{array}{l}X X(X X, X X) \\
X X(X X, X X) \\
X X(X X, X X) \\
X X(X X, X X) \\
X X(X X, X X) \\
X X(X X, X X)\end{array}$ \\
\hline $\begin{array}{l}\text { CSF microbiological tests } \\
\text { - Positive ZN stain } \\
\text { - Positive GeneXpert MTB/RIF } \\
\text { - Positive GeneXpert MTB/RIF UItra } \\
\text { - Positive mycobacterial culture }\end{array}$ & $\begin{array}{l}X X \\
X X \\
X X \\
X X\end{array}$ & $\begin{array}{l}X X(X X \%) \\
X X(X X \%) \\
X X(X X \%) \\
X X(X X \%)\end{array}$ & $\begin{array}{l}X X \\
X X \\
X X \\
X X\end{array}$ & $\begin{array}{l}X X(X X \%) \\
X X(X X \%) \\
X X(X X \%) \\
X X(X X \%)\end{array}$ \\
\hline $\begin{array}{l}\text { Duration of anti-tuberculosis chemotherapy before } \\
\text { enrolment (days) }\end{array}$ & $x X$ & $X X(X X, X X)$ & $x X$ & $X X(X X, X X)$ \\
\hline $\begin{array}{l}\text { Enrolment anti-tuberculosis chemotherapy regimen } \\
\text { - Rifampicin } \\
\text { - Isoniazid } \\
\text { - Pyrazinamide } \\
\text { - Ethambutol } \\
\text { - Streptomycin }\end{array}$ & $\begin{array}{l}X X \\
X X \\
X X \\
X X \\
X X\end{array}$ & $\begin{array}{l}X X(X X \%) \\
X X(X X \%) \\
X X(X X \%) \\
X X(X X \%) \\
X X(X X \%)\end{array}$ & $\begin{array}{l}X X \\
X X \\
X X \\
X X \\
X X\end{array}$ & $\begin{array}{l}X X(X X \%) \\
X X(X X \%) \\
X X(X X \%) \\
X X(X X \%) \\
X X(X X \%)\end{array}$ \\
\hline $\begin{array}{l}\text { Anti-tuberculosis drug resistance* } \\
\text { - Multi-drug resistant or rifampicin mono-resistant } \\
\text { - Isoniazid resistant non-MDR } \\
\text { - Pre-XDR } \\
\text { - XDR } \\
\text { - No or other resistance }\end{array}$ & $\begin{array}{l}X X \\
X X \\
X X \\
X X \\
X X\end{array}$ & $\begin{array}{l}X X(X X \%) \\
X X(X X \%) \\
X X(X X \%) \\
X X(X X \%) \\
X X(X X \%)\end{array}$ & $\begin{array}{l}X X \\
X X \\
X X \\
X X \\
X X\end{array}$ & $\begin{array}{l}X X(X X \%) \\
X X(X X \%) \\
X X(X X \%) \\
X X(X X \%) \\
X X(X X \%)\end{array}$ \\
\hline HIV infection: new diagnosis or known positive status & $X X$ & $X X(X X \%)$ & $X X$ & $X X(X X \%)$ \\
\hline $\begin{array}{l}\text { ART status at enrolment } \\
\text { - ART naïve } \\
\text { - } \leq 3 \text { months of ART } \\
\text { - >3 months of ART } \\
\text { - Unknown } \\
\text { - Undetermined }\end{array}$ & $\begin{array}{l}X X \\
X X \\
X X \\
X X \\
X X\end{array}$ & $\begin{array}{l}X X(X X \%) \\
X X(X X \%) \\
X X(X X \%) \\
X X(X X \%) \\
X X(X X \%)\end{array}$ & $\begin{array}{l}X X \\
X X \\
X X \\
X X \\
X X\end{array}$ & $\begin{array}{l}X X(X X \%) \\
X X(X X \%) \\
X X(X X \%) \\
X X(X X \%) \\
X X(X X \%)\end{array}$ \\
\hline Time to starting ART ${ }^{\#}$ & $x X$ & $X X(X X, X X)$ & $X X$ & $X X(X X, X X)$ \\
\hline Enrolment CD4 count (per mm³) & $x X$ & $X X(X X, X X)$ & $x X$ & $X X(X X, X X)$ \\
\hline
\end{tabular}




\begin{tabular}{|c|c|c|c|c|}
\hline \multirow[t]{2}{*}{ Characteristic } & \multicolumn{2}{|c|}{$\begin{array}{l}\text { Dexamethasone } \\
(\mathrm{N}=\mathrm{XX})\end{array}$} & \multicolumn{2}{|c|}{$\begin{array}{l}\text { Placebo } \\
(\mathrm{N}=\mathrm{XX})\end{array}$} \\
\hline & $\mathbf{N}$ & $\begin{array}{l}\text { Summary } \\
\text { statistic }\end{array}$ & $\mathbf{N}$ & $\begin{array}{l}\text { Summary } \\
\text { statistic }\end{array}$ \\
\hline Enrolment CD4 count (per $\mathrm{mm}^{3}$ ) & $X X$ & $X \times(X \times 0 \%)$ & $y X$ & $X X(X X 0)$ \\
\hline$-51-100$ & $x X$ & $X X(X X \%)$ & $x X$ & $X X(X X \%)$ \\
\hline$-101-200$ & $x X$ & $X X(X X \%)$ & $x X$ & $X X(X X \%)$ \\
\hline - >200 & $X X$ & $X X(X X \%)$ & $X X$ & $X X(X X \%)$ \\
\hline HIV viral load (copies/mL) & $X X$ & $X X(X X, X X)$ & $X X$ & $X X(X X, X X)$ \\
\hline
\end{tabular}

*Results given for sub-group with positive mycobacterial culture on baseline CSF. Pre-XDR and XDR are defined following World Health Organisation definitions ${ }^{11}$. "Results given for sub-group naïve to ART at enrolment.

$\mathrm{N}=$ number of patients included in that statistic. Summary statistic $=$ the median $\left(1^{\text {st }}\right.$ and $3^{\text {rd }}$ quartile) value for continuous data, and the number and frequency (\%) of patients with the characteristic for categorical data.

Definite TBM = positive acid fast bacilli (AFB) on CSF Ziehl Neelsen stain, or positive CSF TB GeneXpert test, OR positive CSF TB culture. Probable or possible TBM defined following uniform case defintion ${ }^{10}$. Confirmed non-TBM = microbiologically confirmed other brain infection. Confirmed additional brain infection includes positive CSF India Ink stain, OR CSF cryptococcal antigen, OR positive blood cryptococcal antigen, OR positive CSF bacterial Gram stain, OR positive CSF bacterial culture, OR positive CSF viral or helminth PCR test.

ART status of a patient will be unknown if i) they are on ART treatment and the start ART date is missing completely; or ii) their ART status is unknown. ART status of a patient will be undetermined if start ART date is present but distinction between $\leq 3$ months of ART, and $>3$ months of ART, cannot be made due to limited date information.

ART=antiretroviral therapy. HTD=Hospital for Tropical Diseases. MRC=Modified Research Council. PNT=Pham Ngoc Thach Hospital for Tuberculosis and Lung Disease. TBM=tuberculous meningitis. XDR=Extensively drug resistant. Xpert=Gene Xpert MTB/RIF. ZN=Ziehl Neelsen.

\section{Table 3. Hazard ratio for death (from any cause) over the first $\mathbf{1 2}$ months after randomisation.}

\begin{tabular}{|l|l|l|l|l|}
\hline & \multicolumn{2}{|c|}{ No. of deaths } & $\begin{array}{l}\text { Hazard ratio } \\
(\mathbf{9 5 \%} \text { CI); } \mathbf{p} \text { value }\end{array}$ & $\begin{array}{l}\text { Test for } \\
\text { proportional } \\
\text { hazards }\end{array}$ \\
\cline { 2 - 6 } & Dexamethasone & Placebo & & \\
\hline All patients & $X X / X X$ & $X X / X X$ & $X . X X(X . X X, X . X X)$ & $X . X X$ \\
Corrected for modified MRC grade & & & $X . X X(X . X X, X . X X)$ & $X . X X$ \\
\hline
\end{tabular}

The primary endpoint will be overall survival, i.e., time from randomisation to death, over the first 12 months of follow-up. This table reports the results from the Cox proportional hazards regression model. The primary effect measure will be the resulting hazard ratio comparing dexamethasone vs. placebo with a corresponding two-sided $95 \%$ confidence interval. In "All patients", treatment will be the only covariate. We will additionally report the hazard ratio if the modified MRC grade is included as stratum variable. The test for proportional hazards will use the Kaplan-Meier as time transformation.

MRC=Modified Research Council

and report the treatment effect by fitting a separate Cox model per subgroup. We will additionally fit a Cox model that includes CD4 count at enrollment as a continuous variable via a restricted cubic spline with four knots and an interaction term with treatment arm.

Kaplan-Meier plots and explicit survival estimates at 3, 6, and 12 months of follow-up will be calculated for the full sample and in the subgroups defined above. We will compare the treatment arms via the absolute risk of death at 12 months (using a Wald-type test based on Kaplan-Meier estimates at 12 months and associated standard errors using Greenwood's formula) and the restricted mean survival until 12 months (using the survRM2 $2^{12}$ package in $\mathrm{R}$ ).

\section{Secondary endpoints}

Secondary outcomes 2 to 7 below are time-to-event outcomes. For each, we will compute and plot the Kaplan-Meier estimates (outcomes 2,4) or the competing risks Aalen-Johansen estimates (outcomes 3, 5, 6 and 7) and report the values of the 
Table 4. Hazard ratios for death (from any cause) by sub-group over the first 12 months after randomisation.

\begin{tabular}{|c|c|c|c|c|c|}
\hline & \multicolumn{2}{|c|}{ No. of deaths } & \multirow{2}{*}{$\begin{array}{l}\text { Hazard ratio } \\
(95 \% \mathrm{CI}) ; p \text { value }\end{array}$} & \multirow{2}{*}{$\begin{array}{l}\text { Test for } \\
\text { proportional } \\
\text { hazards }\end{array}$} & \multirow{2}{*}{$\begin{array}{l}\text { p-value for } \\
\text { heterogeneity* }\end{array}$} \\
\hline & Dexamethasone & Placebo & & & \\
\hline $\begin{array}{l}\text { Modified MRC grade: } \\
\text { - Grade I } \\
\text { - Grade II } \\
\text { - Grade III }\end{array}$ & $\begin{array}{l}X X / X X \\
X X / X X \\
X X / X X\end{array}$ & $\begin{array}{l}X X / X X \\
X X / X X \\
X X / X X\end{array}$ & $\begin{array}{l}X . X X(X . X X, X . X X) \\
X . X X(X . X X, X . X X) \\
X . X X(X . X X, X . X X)\end{array}$ & $\begin{array}{l}X . X X \\
X . X X \\
X . X X\end{array}$ & $X . X X$ \\
\hline $\begin{array}{l}\text { Diagnostic category } \\
\text { - Definite TBM } \\
\text { - Probable TBM } \\
\text { - Possible TBM } \\
\text { - Not TBM }\end{array}$ & $\begin{array}{l}X X / X X \\
X X / X X \\
X X / X X \\
X X / X X\end{array}$ & $\begin{array}{l}X X / X X \\
X X / X X \\
X X / X X \\
X X / X X\end{array}$ & $\begin{array}{l}X . X X(X . X X, X . X X) \\
X . X X(X . X X, X . X X) \\
X . X X(X . X X, X . X X) \\
X . X X(X . X X, X . X X)\end{array}$ & $\begin{array}{l}X . X X \\
X . X X \\
X . X X \\
X . X X\end{array}$ & $X . X X$ \\
\hline $\begin{array}{l}\text { Leukotriene A4 hydrolase } \\
\text { - TT } \\
\text { - CT } \\
\text { - CC }\end{array}$ & $\begin{array}{l}X X / X X \\
X X / X X \\
X X / X X\end{array}$ & $\begin{array}{l}X X / X X \\
X X / X X \\
X X / X X\end{array}$ & $\begin{array}{l}X . X X(X . X X, X . X X) \\
X . X X(X . X X, X . X X) \\
X . X X(X . X X, X . X X)\end{array}$ & $\begin{array}{l}X . X X \\
X . X X \\
X . X X\end{array}$ & $X . X X$ \\
\hline $\begin{array}{l}\text { Anti-tuberculosis drug resistance } \\
\text { - Multi-drug resistant or rifampicin } \\
\text { mono-resistant } \\
\text { - Isoniazid resistant non-MDR } \\
\text { - Pre-XDR } \\
\text { - XDR } \\
\text { - No or other resistance }\end{array}$ & $\begin{array}{l}X X \\
X X \\
X X \\
X X \\
X X\end{array}$ & $\begin{array}{l}X X(X X \%) \\
X X(X X \%) \\
X X(X X \%) \\
X X(X X \%) \\
X X(X X \%)\end{array}$ & $\begin{array}{l}X X \\
X X \\
X X \\
X X \\
X X\end{array}$ & $\begin{array}{l}X . X X \\
X . X X \\
X . X X \\
X . X X \\
X . X X\end{array}$ & $X . X X$ \\
\hline $\begin{array}{l}\text { ART status at enrolment } \\
\text { - ART naïve } \\
-\leq 3 \text { months of ART } \\
->3 \text { months of ART }\end{array}$ & $\begin{array}{l}X X / X X \\
X X / X X \\
X X / X X\end{array}$ & $\begin{array}{l}X X / X X \\
X X / X X \\
X X / X X\end{array}$ & $\begin{array}{l}X . X X(X . X X, X . X X) \\
X . X X(X . X X, X . X X) \\
X . X X(X . X X, X . X X)\end{array}$ & $\begin{array}{l}X . X X \\
X . X X \\
X . X X\end{array}$ & $X . X X$ \\
\hline $\begin{array}{l}\text { Enrolment CD4 count }\left(\text { per } \mathrm{mm}^{3}\right) \\
-<50 \\
-51-100 \\
-101-200 \\
->200\end{array}$ & $\begin{array}{l}X X / X X \\
X X / X X \\
X X / X X \\
X X / X X\end{array}$ & $\begin{array}{l}X X / X X \\
X X / X X \\
X X / X X \\
X X / X X\end{array}$ & $\begin{array}{l}X . X X(X . X X, X . X X) \\
X . X X(X . X X, X . X X) \\
X . X X(X . X X, X . X X) \\
X . X X(X . X X, X . X X)\end{array}$ & $\begin{array}{l}X . X X \\
X . X X \\
X . X X \\
X . X X\end{array}$ & $X . X X$ \\
\hline
\end{tabular}

*Heterogeneity will be tested with a Cox regression model that includes an interaction between treatment effect and subgroup. "Results will be given for sub-group with positive mycobacterial culture on baseline CSF. Pre-XDR and XDR are defined following World Health Organisation definitions ${ }^{11}$.

The primary endpoint will be overall survival, i.e., time from randomisation to death, over the first 12 months of follow-up. This table reports the results from the Cox proportional hazards regression model. The primary effect measure will be the resulting hazard ratio comparing dexamethasone vs. placebo with a corresponding two-sided 95\% confidence interval.

In these subgroup analyses, a separate Cox model will be fitted for each value of the subgroup. The "Test for heterogeneity" will be based on the likelihood ratio test that includes subgroup as covariate and compares the models with subgroup as main effect only and with subgroup as treatment effect modifier, with TBM MRC severity grade at enrolment (I, II, or III) as covariates.

ART=antiretroviral therapy. MRC=Modified Research Council. TBM=tuberculous meningitis. XDR=extensively drug resistant.

estimates at 3,6 and 12 months. We will also fit a Cox proportional hazards model (results are interpreted as relative cause-specific hazards in the presence of competing risks). Analyses will be performed for the full samples (ITT and PP). Subgroup analyses are specified below per secondary endpoint. For the subgroup analyses, we follow the same procedure as for the primary outcome: we fit separate models per subgroup and we fit a model in which we test for interaction by subgroup.

\section{Neurological disability at 12 months from randomisation}

Neurological disability will be assessed by the modified Rankin score at months $1,2,3,6,9$, and 12 from randomisation (Table 5). The main secondary endpoint is the 12-month assessment and subjects who died before 12 months will be treated as having a score of 6 ('dead'). Rankin score assessments will be included from clinical assessments performed monthly 
Table 5. The Modified Rankin Scale.

\begin{tabular}{|l|l|}
\hline Score & Description \\
\hline 0 & No symptoms \\
\hline 1 & Minor symptoms not interfering with lifestyle \\
\hline 2 & Symptoms that lead to some restriction in lifestyle, but do not interfere with the patients ability to look after themselves \\
\hline 3 & Symptoms that restrict lifestyle and prevent totally independent living \\
\hline 4 & Symptoms that clearly prevent independent living, although the patient does not need constant care and attention. \\
\hline 5 & Totally dependent, requiring constant help day and night. \\
\hline 6 & Death \\
\hline
\end{tabular}

(+/- 7 days), with the exception of the 12-month clinical assessment for which an acceptable range of -10 days/ +1 month will be applied. All clinical assessment timings are based on days from randomisation (i.e., 'day 0 ' is labeled as the first day study drug is received, with study drug received immediately after randomisation).

Neurological disability (as assessed by the ordinal modified Rankin scale) at 12 months will be compared between the two arms with a proportional odds logistic regression model with the treatment assignment as the main covariate and adjustment for TBM MRC severity grade. The result will be summarised as a cumulative odds ratio with corresponding $95 \%$ confidence interval and $\mathrm{p}$-value. Individuals who withdrew or were lost to follow-up before 12 months are excluded.

\section{First new neurological event or death over the first 12} months after randomisation

A new neurological event is defined as a fall in GCS by $\geq 2$ points for $\geq 2$ days from the highest previously recorded GCS (including baseline) or the onset of any of the following clinical adverse events: cerebellar symptoms, focal neurological signs, or onset of seizures. A description of all the events will be given, summarising how they met the criteria.

Analyses will additionally be performed in the subgroups defined by TBM MRC severity grade, diagnostic category, ART status at enrolment, time from randomisation to start of ART, and baseline CD4 count.

\section{First IRIS event over the first 6 months after randomisation}

The criteria for neurological IRIS diagnosis is defined in the study protocol ${ }^{1}$. The neurological IRIS rate will be defined as the number of IRIS events divided by the observed person-time of follow-up in each treatment group. A description of all the IRIS events will be given, describing how they met the IRIS diagnostic criteria.

The number of IRIS events in each group will be summarised. Death will be interpreted as a competing risk. Analyses will additionally be performed in the subgroups defined by TBM MRC grade, diagnostic category, ART status at enrolment, time from randomisation to start of ART, and baseline CD4 count.

4. AIDS-defining event or death over the first 12 months after randomisation

Acquired immunodeficiency syndrome (AIDS)-defining illnesses will be defined as per the World Health Organisation (WHO) classification. WHO define AIDS as a clinical diagnosis (presumptive or definitive) of any stage 4 HIV condition ${ }^{13}$.

\section{HIV-associated malignancy over the first 12 months} after randomisation

HIV-associated malignancy is defined as new diagnosis of one or more of the three major HIV-associated malignancies; Kaposi sarcoma, high grade B-cell non-Hodgkin lymphoma or invasive cervical cancer.

The number of HIV-associated malignancy events in each group will be summarised. Death will be interpreted as a competing risk. Analyses will additionally be performed in the subgroups defined by ART status at enrolment.

\section{Use of open-label corticosteroid treatment for any} reason, and at any time, after randomisation

The number of open-label corticosteroid treatment events in each group will be summarised. The reasons for corticosteroid treatment will be listed. Death will be interpreted as a competing risk.

\section{Requirement for shunt surgery by 12 months}

The number of shunts performed in each group will be summarised. Death will be interpreted as a competing risk.

\section{Adverse events until 12 months}

Serious adverse events (SAE) are defined in the study protocol $^{1}$. SAE will be sub-grouped into categories. SAE will be grouped and graded as per Common Terminology Criteria for Adverse Events (CTCAE) ${ }^{14}$. 
The number of patients with any serious adverse event will be summarised and compared between the two treatment arms based on the chi-squared test, or Fisher's exact test in case the expected count under the null hypothesis in at least one of the cells is smaller than one ${ }^{15}$. Specific adverse events will be summarised, but not formally compared. The total number of serious adverse event episodes per patient will also be summarised and informally compared based on a quasi-Poisson regression model with treatment as the only covariate.

The following subgroups of adverse events will also be separately summarised: clinical grade $3 \& 4$ adverse events; serious adverse events possibly, probably, or definitely related to the study drug; adverse events leading to TB treatment or ARV interruptions. Grade $3 \& 4$ laboratory abnormalities will be summarised in the same way as clinical adverse events. Adverse events will be shown as per Table 6-Table10.

Explanatory analysis via multivariate model

A multivariate model of variables independently associated with death will include: age, enrolment GCS, TBM diagnostic category (definite, probable, possible), study drug allocation (dexamethasone vs. placebo), CD4 count, CSF total leucocytes, CSF total neutrophils, CSF blood:glucose ratio, CSF

Table 6a. Summary of serious adverse events.

\begin{tabular}{|l|l|l|l|l|l|}
\hline Characteristic & $\begin{array}{l}\text { Dexamethasone } \\
(\mathbf{N = X X )}\end{array}$ & $\begin{array}{l}\text { Placebo } \\
(\mathbf{N}=\mathbf{X X})\end{array}$ & $\begin{array}{l}\text { Comparison } \\
\text { (p-value) }\end{array}$ \\
\hline & $\mathbf{N . p t}$ & N.ae & N.pt & N.ae & \\
\hline Any selected serious adverse event & $X X(X X \%)$ & $X X$ & $X X(X X \%)$ & $X X$ & $X . X X$ \\
\hline Serious adverse events of all types & $X X(X X \%)$ & $X X$ & $X X(X X \%)$ & $X X$ & $X . X X$ \\
\hline
\end{tabular}

$N . p t=$ the number of patients with at least one serious adverse event (\% of all patients receiving the same intervention)

$N \cdot a e=$ the total number of episodes of that particular serious adverse event

Table $6 \mathrm{~b}$. Summary of serious adverse events, shown by reasons for which they were considered serious, not shown by study arm.

\begin{tabular}{|c|c|c|c|c|c|c|}
\hline & $\begin{array}{l}\text { Causes } \\
\text { death } \\
(\mathrm{N}=\mathrm{XX})\end{array}$ & $\begin{array}{l}\text { Life } \\
\text { threatening } \\
\text { event* } \\
(\mathrm{N}=\mathrm{XX})\end{array}$ & $\begin{array}{l}\text { Hospitalisation of } \\
\text { prolongation of } \\
\text { hospitalisation } \\
(\mathrm{N}=\mathrm{XX})\end{array}$ & $\begin{array}{l}\text { Persistent or } \\
\text { significant } \\
\text { disability/ } \\
\text { incapacity** } \\
(\mathrm{N}=\mathrm{XX})\end{array}$ & $\begin{array}{l}\text { Congenital } \\
\text { anomaly/ } \\
\text { birth defect } \\
(\mathrm{N}=\mathrm{XX})\end{array}$ & $\begin{array}{l}\text { Important medical event } \\
\text { which may jeopardise } \\
\text { the patient and/or } \\
\text { require intervention } \\
(\mathrm{N}=\mathrm{XX})\end{array}$ \\
\hline & $\begin{array}{l}\text { n } \\
\text { Summary } \\
\text { statistic }\end{array}$ & $\begin{array}{l}\text { n } \\
\text { Summary } \\
\text { statistic }\end{array}$ & $\begin{array}{l}\text { n } \\
\text { Summary statistic }\end{array}$ & $\begin{array}{l}\text { n } \\
\text { Summary statistic }\end{array}$ & $\begin{array}{l}\text { n } \\
\text { Summary } \\
\text { statistic }\end{array}$ & $\begin{array}{l}\text { n } \\
\text { Summary } \\
\text { statistic }\end{array}$ \\
\hline Name of event & $X X(X X \%)$ & $X X(X X \%)$ & $X X(X X \%)$ & $X X(X X \%)$ & $X X(X X \%)$ & $X X(X X \%)$ \\
\hline \multicolumn{7}{|c|}{$\begin{array}{l}\text { * Subjects were at immediate risk of death at the time of the event; it does not refer to an event which hypothetically might have caused death if it were } \\
\text { more severe }\end{array}$} \\
\hline
\end{tabular}

Table 7. Summary of serious adverse events possibly, probably, or definitely related to the study drug.

\begin{tabular}{|c|c|c|c|c|c|}
\hline \multirow[t]{2}{*}{ Characteristic } & \multicolumn{2}{|c|}{$\begin{array}{l}\text { Dexamethasone } \\
(\mathrm{N}=\mathrm{XX})\end{array}$} & \multicolumn{2}{|l|}{$\begin{array}{l}\text { Placebo } \\
(N=X X)\end{array}$} & \multirow[t]{2}{*}{$\begin{array}{l}\text { Comparison } \\
\text { (p-value) }\end{array}$} \\
\hline & N.pt & N.ae & N.pt & N.ae & \\
\hline $\begin{array}{l}\text { Any selected serious adverse event possibly, probably, } \\
\text { or definitely related to the study drug }\end{array}$ & $X X(X X \%)$ & $X X$ & $X X(X X \%)$ & $X X$ & $X . X X$ \\
\hline $\begin{array}{l}\text { Serious adverse events of all types possibly, probably, } \\
\text { or definitely related to the study drug }\end{array}$ & $X X(X X \%)$ & $X X$ & $X X(X X \%)$ & $X X$ & $X . X X$ \\
\hline
\end{tabular}


Table 8 . Summary of clinical grade $3 \& 4$ adverse events.

\begin{tabular}{|l|l|l|l|l|l|}
\hline Characteristic & $\begin{array}{l}\text { Dexamethasone } \\
(\mathbf{N = X X )}\end{array}$ & $\begin{array}{l}\text { Placebo } \\
(\mathbf{N = X X )}\end{array}$ & $\begin{array}{l}\text { Comparison } \\
\text { (p-value) }\end{array}$ \\
\hline & N.pt & N.ae & N.pt & N.ae & \\
\hline Any selected clinical grade 3\&4 adverse event & $X X(X X \%)$ & $X X$ & $X X(X X \%)$ & $X X$ & $X . X X$ \\
\hline Clinical grade 3 adverse events of all types & $X X(X X \%)$ & $X X$ & $X X(X X \%)$ & $X X$ & $X . X X$ \\
\hline Clinical grade 4 adverse events of all types & $X X(X X \%)$ & $X X$ & $X X(X X \%)$ & $X X$ & $X . X X$ \\
\hline Clinical grade 3\&4 adverse events of all types & $X X(X X \%)$ & $X X$ & $X X(X X \%)$ & $X X$ & $X . X X$ \\
\hline
\end{tabular}

$\mathrm{N} . \mathrm{pt}=$ the number of patients with at least one adverse event (\% of all patients receiving the same intervention)

$\mathrm{N} . \mathrm{ae}=$ the total number of episodes of that particular adverse event

The 'clinical grade $3 \& 4$ adverse events of all types' grouping will include only the group of adverse events that cannot be separated into grades 3 or 4 (recorded as 'grade 3 or $4^{\prime}$ at the beginning of the trial). These events will not also be represented in the above 'grade 3 ' and 'grade 4' rows.

Table 9. Summary of adverse events leading to TB treatment or antiretroviral (ARV) interruptions.

\begin{tabular}{|c|c|c|c|c|c|}
\hline \multirow[t]{2}{*}{ Characteristic } & \multicolumn{2}{|c|}{$\begin{array}{l}\text { Dexamethasone } \\
(\mathrm{N}=\mathrm{XX})\end{array}$} & \multicolumn{2}{|l|}{$\begin{array}{l}\text { Placebo } \\
(\mathrm{N}=\mathrm{XX})\end{array}$} & \multirow[t]{2}{*}{$\begin{array}{l}\text { Comparison } \\
\text { (p-value) }\end{array}$} \\
\hline & N.pt & N.ae & N.pt & N.ae & \\
\hline Any selected adverse event leading to TB treatment or antiretroviral interruptions & $X X(X X \%)$ & $X X$ & $X X(X X \%)$ & $X X$ & $X . X X$ \\
\hline Adverse events of all types leading to TB treatment or antiretroviral interruptions & $X X(X X \%)$ & $X X$ & $X X(X X \%)$ & $x X$ & $X . X X$ \\
\hline
\end{tabular}

N.pt = the number of patients with at least one serious adverse event (\% of all patients receiving the same intervention)

$\mathrm{N} \cdot \mathrm{ae}=$ the total number of episodes of that particular serious adverse event

Table 10. Summary of Grade $\mathbf{3} \& 4$ laboratory abnormalities.

\begin{tabular}{|l|l|l|l|l|l|}
\hline Characteristic & $\begin{array}{l}\text { Dexamethasone } \\
(\mathbf{N}=\mathbf{X X})\end{array}$ & $\begin{array}{l}\text { Placebo } \\
(\mathbf{N}=\mathbf{X X )}\end{array}$ & & $\begin{array}{l}\text { Comparison } \\
\text { (p-value) }\end{array}$ \\
\hline & $\mathbf{N . p t}$ & $\mathbf{N . a e}$ & $\mathbf{N . p t}$ & $\mathbf{N . a e}$ & \\
\hline Any selected grade 3\&4 laboratory abnormalities & $X X(X X \%)$ & $X X$ & $X X(X X \%)$ & $X X$ & $X . X X$ \\
\hline Grade 3\&4 laboratory abnormalities of all types & $X X(X X \%)$ & $X X$ & $X X(X X \%)$ & $X X$ & $X . X X$ \\
\hline
\end{tabular}

$N . p t=$ the number of patients with at least one serious adverse event (\% of all patients receiving the same intervention)

$\mathrm{N} . \mathrm{ae}=$ the total number of episodes of that particular serious adverse event

lactate. Numeric variables will be analysed using restricted cubic splines. We will not include any interactions.

\section{Data availability}

No data is associated with this article.

\section{Acknowledgments}

The authors would like to acknowledge the ACT HIV investigators. A full list of ACT HIV investigators appears in Box 1. Additionally the authors would like to acknowledge the ACT HIV steering committee (TSC); Dr Truong Huu Khanh (Infectious Diseases Physician, Paediatric Hospital Number 1, Ho Chi Minh City, Vietnam), Professor Ben Marais (Senior
Tuberculosis Researcher and Trialist, University of Sydney, Australia), Professor Nicholas Paton (Infectious Diseases Physician and Clinical Trialist, National University of Singapore, Singapore), and TSC Chair Professor Robert Wilkinson (Honorary Professor and Director Wellcome Centre for Infectious Diseases Research in Africa, University of Cape Town, South Africa), and the ACT HIV data monitoring committee (DMC); Professor Graeme Meintjes (Senior Infectious Diseases/HIV Physician, University of Cape Town, South Africa), Professor Nina Ruslami (Senior TBM Clinician and Researcher, Universitas Padjadjaran, Bandung, Indonesia), and DMC Chair Professor Sarah Walker (Senior Statistician and Clinical Trialist, MRC Clinical Trials Unit, University College London). 
Box 1. ACT HIV study group (listed by group, and then alphabetically).

Pham Ngoc Thach Hospital for Tuberculosis and Lung Disease, Ho Chi Minh City, Vietnam

\begin{tabular}{|c|c|c|}
\hline $\begin{array}{l}\text { Dr Truong Thi Anh } \\
\text { Dr Nguyen Duc Bang } \\
\text { Dr Luu Tuan Bang } \\
\text { Pham Thi Anh Dao } \\
\text { Dr Nguyen Thuong Dat } \\
\text { Do Kim Dung } \\
\text { Dr Le Tien Dung }\end{array}$ & $\begin{array}{l}\text { Dr Dang Minh Duong } \\
\text { Dr Do Chau Giang } \\
\text { Dr Dang Thi Minh Ha } \\
\text { Dr Nguyen Thi Thu Hiep } \\
\text { Dr Nguyen Huu Lan } \\
\text { Dr Nguyen Huu Minh } \\
\text { Dr Huynh Phat }\end{array}$ & $\begin{array}{l}\text { Dr Dang Van Thanh } \\
\text { Vo Thi Thanh Thuy } \\
\text { Dr Nghiem Huyen Trang } \\
\text { Dr Nguyen Nang Vien }\end{array}$ \\
\hline
\end{tabular}

Hospital for Tropical Diseases, Ho Chi Minh City, Vietnam
Dr Le Thi My Chau
Dr Tran Minh Luan
Dr Van Thi Xuan Quynh
Dr Nguyen Van Vinh Chau
Dr Ho Dang Trung Nghia
Nguyen Thi Ha
Dr Pham Kieu Nguyet Oanh
Dr Nguyen Ho Hong Hanh
Dr Nguyen Hoan Phu
Dr Nguyen Truc Thanh

\section{Oxford University Clinical Research Unit, Ho Chi Minh City, Vietnam}

\begin{tabular}{l|l|l} 
Professor Guy E. Thwaites & Lam Hong Bao Ngoc & Dr Joseph Donovan \\
Biostatistics group & Nguyen Thi Thao Nguyen & Vu Thi Mong Dung \\
\hline Dr Ronald B. Geskus & Trinh Khanh Phuong & Vu Thi Ngoc Ha \\
Dr Dong Huu Khanh Trinh & Dr Nguyen Than Ha Quyen & Pham Thi Kim \\
Dr Le Thi Phuong Thao & Nguyen Bao Tran & Nguyen Le Quang \\
Clinical trials unit & Nguyen Thi Huyen Trang & Do Dang Anh Thu \\
\hline Dr Nguyen Thi Phuong Dung & Dr Celine Vidaillac & Dr Nguyen Thuy Thuong \\
Dau Thi Thanh Huong & Tuberculosis group & Thuong \\
Evelyne Kestelyn & Tran Dinh Dinh & Dr Trinh Thi Bich Tram
\end{tabular}

Cipto Mangunkusumo Hospital, Jakarta, Indonesia

Dr Putri Widya Andini

Dr Riwanti Estiasari

Dr Ardiana Kusumaningrum

Dr Darma Imran

Dr David Pangeran

Dr Cakra Parindra Gasmara

Dr Kartika Maharani

Dr Maria Gabriella Sainlia

Persahabatan Hospital, Jakarta, Indonesia

\begin{tabular}{|l|l|l|}
\hline Dr Erlina Burhan & Dr Ihsan Hamdani & Dr Muhammad Fakhri Zahir \\
Dr Teuku Reyhan Gamal & Dr Ashwin Marcel Rumawas &
\end{tabular}

Eijkman-Oxford Clinical Research Unit, Jakarta, Indonesia

Professor J. Kevin Baird

Ichsan Kalbuadi

Lia Waslia

Professor Reinout van Crevel

Mugi Nursatya

Anna Laksmi Sri Wardhani

Dr Raph L. Hamers

Mutia Rahardjani

Jeny

Dr Decy Subekti

1. Donovan J, Phu NH, Mai NTH, et al:: Adjunctive dexamethasone for the treatment of HIV-infected adults with tuberculous meningitis (ACT HIV): Study protocol for a randomised controlled trial [version 2; peer review: 1 approved, 2 approved with reservations]. Wellcome Open Res. 2018; 3: 31. PubMed Abstract | Publisher Full Text | Free Full Text

2. Christensen ASH, Roed C, Omland LH, et al.: Long-term mortality in patients with tuberculous meningitis: a Danish nationwide cohort study. PLOS One. 2011; 6(11): e27900.

PubMed Abstract | Publisher Full Text | Free Full Text
3. Vinnard C, King L, Munsiff S, et al.: Long-term Mortality of Patients With Tuberculous Meningitis in New York City: A Cohort Study. Clin Infect Dis. 2017; 64(4): 401-407.

PubMed Abstract | Publisher Full Text | Free Full Text

4. Thwaites GE, Nguyen DB, Nguyen HD, et al.: Dexamethasone for the Treatment of Tuberculous Meningitis in Adolescents and Adults. N Eng/J Med. 2004; 351(17): 1741-51.
PubMed Abstract | Publisher Full Text

5. Ruslami R, Ganiem AR, Dian S, et al.: Intensified regimen containing 
rifampicin and moxifloxacin for tuberculous meningitis: an open-label, randomised controlled phase 2 trial. Lancet Infect Dis. 2013; 13(1): 27-35. PubMed Abstract | Publisher Full Text

6. Davis AG, Donovan J, Bremer M, et al.: Host Directed Therapies for Tuberculous Meningitis [version 2; peer review: 1 approved, 1 approved with reservations]. Wellcome Open Res. 2020; 5: 292. Publisher Full Text

7. Thuong NTT, Heemskerk D, Tram TTB, et al.: Leukotriene A4 Hydrolase Genotype and HIV Infection Influence Intracerebral Inflammation and Survival From Tuberculous Meningitis. J Infect Dis. 2017; 215(7): 1020-1028. PubMed Abstract | Publisher Full Text | Free Full Text

8. Wilkinson RJ, Rohlwink U, Misra UK, et al.: Tuberculous meningitis. Nat Rev Neurol. 2017; 13(10): 581-98. PubMed Abstract | Publisher Full Text

9. R Foundation for Statistical Computing, Vienna A. R Core Team: R: A language and environment for statistical computing. 2021. Reference Source

10. Marais S, Thwaites G, Schoeman JF, et al.: Tuberculous meningitis: a uniform case definition for use in clinical research. Lancet Infect Dis. 2010; 10(11):
803-12.

PubMed Abstract | Publisher Full Text

11. World Health Organization: WHO announces updated definitions of extensively drug-resistant tuberculosis. Geneva. 2021; (accessed July 30, 2021). Reference Source

12. Uno H, Tian L, Horiguchi M, et al.: survRM2: Comparing Restricted Mean Survival Time. R package version 1.0-3. 2020. Reference Source

13. World Health Organization: WHO Case Definitions Of HIV For Surveillance And Revised Clinical Staging And Immunological Classification Of HIVRelated Disease In Adults And Children HIVIAIDS Programme. Geneva, 2007.

Reference Source

14. National Cancer Institute: Common Terminology Criteria for Adverse Events (CTCAE) v5.0. 2017; (accessed May 27, 2021). Reference Source

15. Campbell I: Chi-squared and Fisher-Irwin tests of two-by-two tables with small sample recommendations. Stat Med. 2007; 26(19): 3661-75. PubMed Abstract | Publisher Full Text 


\section{Open Peer Review}

\section{Current Peer Review Status:}

\section{Version 1}

Reviewer Report 10 November 2021

https://doi.org/10.21956/wellcomeopenres.18947.r46542

(C) 2021 Mwaba P. This is an open access peer review report distributed under the terms of the Creative Commons Attribution License, which permits unrestricted use, distribution, and reproduction in any medium, provided the original work is properly cited.

\section{Peter Mwaba}

Faculty of Medicine, Lusaka Apex Medical University, Lusaka, Zambia

Evidence from Clinical trials that have been done this far on disseminated tuberculosis and in Particular TBM have not been conclusive on the role of Corticosteroids in improving outcomes. Corticosteroids have been tried in Tuberculous pericarditis, granulomatous diseases and Leprosy reactions with good outcomes. Their role in bacterial meningitis particularly WaterhouseFriderichsen Syndrome has been shown to be positive.

Given the debate on BCG vaccine and its possible role in preventing Tuberculous meningitis it would be of value if there is a specific way of confirming the number of participants who have had the vaccine. Some of the Secondary end points may be drug related and it is therefore important to know the exact drug regimen used for both TBM and HIV. Otherwise an excellent piece of work

Is the rationale for, and objectives of, the study clearly described?

Yes

Is the study design appropriate for the research question?

Yes

Are sufficient details of the methods provided to allow replication by others?

Yes

Are the datasets clearly presented in a useable and accessible format?

Yes

Competing Interests: No competing interests were disclosed.

Reviewer Expertise: Infectious disease

I confirm that I have read this submission and believe that I have an appropriate level of 
expertise to confirm that it is of an acceptable scientific standard.

Reviewer Report 01 November 2021

https://doi.org/10.21956/wellcomeopenres.18947.r46541

(C) 2021 Garg R et al. This is an open access peer review report distributed under the terms of the Creative Commons Attribution License, which permits unrestricted use, distribution, and reproduction in any medium, provided the original work is properly cited.

\section{Ravindra Kumar Garg}

Department of Neurology, King George's Medical University, Lucknow, Uttar Pradesh, India

Hardeep Singh Malhotra

Department of Neurology, King George's Medical University, Lucknow, Uttar Pradesh, India

The issue of potential benefits of corticosteroids in HIV-associated TBM remains unaddressed so far. The authors have planned an almost flawless study to address the issue. The authors are trying the same corticosteroids regimen as had been used in earlier trials which included both HIV-negative and HIV-positive TBM patients. A small paragraph detailing the anti-tuberculosis regimen (drugs and duration) and the ART regimen should be added for better clarity. How will the authors address the issue of "paradoxical reaction"? I find vision loss a major disability among TBM survivors, kindly give due attention to this as well.

Regarding analysis, I feel that the use of median should depend on the distribution instead of assuming an asymmetric distribution. We can, therefore, include 'mean' too and look for its statistical relevance.

In my opinion, the corticosteroids regimen that has been planned to use in this study is a bit complicated. With a simpler regimen compliance would be better.

Is the rationale for, and objectives of, the study clearly described?

Yes

Is the study design appropriate for the research question?

Yes

Are sufficient details of the methods provided to allow replication by others?

Yes

Are the datasets clearly presented in a useable and accessible format?

Yes

Competing Interests: No competing interests were disclosed.

Reviewer Expertise: CNS tuberculosis and other CNS infections 
We confirm that we have read this submission and believe that we have an appropriate level of expertise to confirm that it is of an acceptable scientific standard.

Reviewer Report 27 October 2021

https://doi.org/10.21956/wellcomeopenres.18947.r46545

(C) 2021 Nagelkerke $\mathbf{N}$. This is an open access peer review report distributed under the terms of the Creative Commons Attribution License, which permits unrestricted use, distribution, and reproduction in any medium, provided the original work is properly cited.

\section{Nico (Nicolaas) Nagelkerke}

Centre for Global Health Research, Unity Health Toronto, Dalla Lana School of Public Health, University of Toronto, Toronto, ON, Canada

This is slick analysis plan by a clearly highly experienced team for a multi-centre randomized clinical trial following highly standardized methodologies. The proposed analyses will lead to a correct, and generally accepted result, as well as guide the future standard of care guidelines for Tuberculous meningitis in HIV+ patients. As the drug on trial is cheap and easy to administer, guidelines will be applicable globally (including Africa where HIV prevalence is highest).

Is the rationale for, and objectives of, the study clearly described?

Yes

Is the study design appropriate for the research question?

Yes

Are sufficient details of the methods provided to allow replication by others?

Yes

Are the datasets clearly presented in a useable and accessible format?

Not applicable

Competing Interests: No competing interests were disclosed.

Reviewer Expertise: Biostatistics and Infectious disease epidemiology

I confirm that I have read this submission and believe that I have an appropriate level of expertise to confirm that it is of an acceptable scientific standard. 